\title{
Un cycle des phosphoinositides existe dans le noyau
}

Dans la plupart des membranes plasmiques de cellules eucaryotes il existe une voie de transduction du signal qui utilise les inositol lipides comme précurseurs des seconds messagers inositol trisphosphate $\left(\mathrm{IP}_{3}\right)$ et diacylglycérol (DAG). Il est couramment admis que ce métabolisme, connu aussi sous le nom de cycle des phosphoinositides, a pour conséquence une mobilisation calcique à partir des réserves intracellulaires due à l'action de $\mathrm{l}^{\prime} \mathrm{IP}_{3}$ et une activation de la protéine kinase $\mathrm{C}$ (PKC) par les DAG [1]. Le métabolisme des phosphoinositides apparaît aujourd'hui relativement complexe ; en effet, les nombreuses enzymes impliquées possèdent plusieurs isoformes répondant, semble-t-il, à des modes de régulation différents [2]. Le lieu privilégié de ces réactions enzymatiques clés est classiquement envisagé dans la membrane plasmique ou à l'interface membrane/cytosol, mais on s'aperçoit actuellement qu'elles peuvent dépasser ce cadre [3, 4].

Les premières publications rapportant une incorporation de ${ }^{32} \mathrm{P}$ à partir de $\gamma^{32}$ P[ATP] dans les inositol phospholipides présents à l'intérieur du compartiment nucléaire datent d'une dizaine d'années. En 1983, Smith et Wells [5] furent parmi les premiers à démontrer une localisation nucléaire des inositol lipides et à suggérer la présence des enzymes responsables de leur métabolisme dans le noyau d'hépatocytes de rat. En utilisant des cellules érythro-leucémiques de Friend, Cocco et al. [6] ont mis en évidence, dès 1987, des différences d'incorporation de ${ }^{32} \mathrm{P}$ dans les phosphatidylinositol bisphosphates $\left(\mathrm{PIP}_{2}\right)$ de noyau, selon l'état de différenciation des cellules. Peu après, le même groupe observait des modifications nettes du métabolisme des phosphoinositides dans les noyaux de fibroblastes Swiss 3T3 stimulés par l'IGF1 [7], alors que ce fac$m / s n^{\circ} 7$, vol. 8, septembre 92 teur de croissance n'avait aucune action sur des noyaux isolés. Capitani et al. [8] laissaient supposer que les enzymes du métabolisme des phosphoinositides étaient associées aux matrices nucléaires. L'ensemble de ces résultats suggérait de façon indirecte l'existence d'un cycle des phosphoinositides dans le noyau sans toutefois convaincre pleinement la communauté scientifique réticente, notamment à cause d'éventuels problèmes de contaminations pendant la préparation des noyaux.

Cependant, récemment, un nouvel élan a été donné à ces travaux. En effet, le groupe de N. Divecha et $\mathrm{R}$. Irvine a clairement montré qu'un signal initié par un récepteur possédant une activité tyrosine kinase intrinsèque, situé dans la membrane plasmique (le récepteur de l'IGF1), peut contrôler spécifiquement le métabolisme des phosphoinositides dans les noyaux de fibroblastes sans affecter ce métabolisme dans les membranes plasmiques [4].

Divecha et al. ont pu quantifier, en masse, les inositol lipides et les DAG dans les noyaux de fibroblastes Swiss 3T3. Dans des cellules au repos il y aurait à l'intérieur des noyaux isolés en présence de détergents (Triton X-100/désoxycholate de sodium), de l'ordre de $110 \mathrm{pmoles} / \mathrm{mg}$ de protéine de DAG, 2 nmoles/mg de protéine de phosphatidylinositol (PI), 55 pmoles/mg de protéine de phosphatidylinositol 4 phosphate (PI4P) et environ 7 pmoles/mg de protéine de phosphatidylinositol 4,5 bisphosphate $\left(\mathrm{PI} 4,5 \mathrm{P}_{2}\right)$. Les auteurs ont comparé l'effet de l'IGF1 à celui de la bombésine sur le cycle des phosphoinositides dans les fibroblastes Swiss 3T3. La bombésine active clairement la PLC dans la membrane plasmique ou à l'interface membrane/cytosol mais n'a aucun effet sur le métabolisme des phosphoinositides dans le noyau, alors que l'IGF1 n'active pas de PLC au niveau membranaire mais agit spécifiquement sur le cycle des phosphoinositides dans le noyau. En effet, la quantité de DAG dans les noyaux atteint 200 pmoles/mg de protéine après 5 minutes de stimulation par l'IGF1; parallèlement les auteurs observent une chute transitoire de la concentration de $\mathrm{PI} 4,5 \mathrm{P}_{2}$ avant que le stock ne se reconstitue après 15 minutes d'activation. La production de DAG, observée spécifiquement dans le noyau sous l'effet de l'IGF1, est accompagnée d'une translocation d'une isoforme de $80 \mathrm{kDa}$ de la $\mathrm{PKC}$ vers le noyau. D'autres groupes avaient préalablement montré la présence d'une PKC dans le noyau ainsi que la possibilité de sa translocation vers le compartiment nucléaire [9]. A ces observations viennent s'ajouter des résultats récents indiquant que des protéines de liaison du calcium et des phospholipides appelées annexines I et II, sont également présentes dans le noyau $[10,11]$. L'annexine II, substrat des protéine tyrosine kinases, fait partie d'un complexe protéique qui interagit avec l'ADN polymérase $\alpha$ [10]. Enfin, il est intéressant de relier ces résultats avec l'observation de phosphorylations spécifiques sur des résidus de tyrosine de plusieurs protéines nucléaires sous l'effet de certains facteurs de croissance, notamment l'IGF1 [12] (m/s $n^{\circ} 3$, vol. 8, p. 80). Les variations de concentration des inositol lipides et des DAG dans les noyaux sont le reflet d'activités enzymatiques, telles que la PLC et les phosphoinositides kinases. Nous avons effectivement mesuré des activités PIkinase, PIP-kinase, DAG-kinase et PLC dans les noyaux d'hépatocytes de rat et de fibroblastes non stimulés [13].

Collaboration en partie financée par un contrat NWO/INSERM. 
Le noyau est un organite particulièrement bien organisé dans lequel chacune des fonctions spécifiques est localisée dans une région nucléaire spécialisée et non pas distribuée de façon diffuse [14]. Nous avons donc recherché une localisation plus précise des enzymes du métabolisme des phosphoinositides dans les noyaux de fibroblastes et d'hépatocytes de rat. Après traitement des noyaux avec un détergent afin d'éliminer l'enveloppe nucléaire (double bicouche lipidique), puis avec les nucléases (DNase et RNase) et de fortes concentrations en sel, on obtient un réseau protéique classiquement dénommé "matrices nucléaires " [15]. Ces matrices nucléaires peuvent être divisées au moins en deux compartiments, la matrice interne contenant les éléments du nucléole, et la matrice périphérique ou lamina (lamines A, B et $\mathrm{C}$ ) contenant les pores nucléaires. Plusieurs enzymes impliquées dans la réplication et la transcription sont associées aux matrices nucléaires.

Grâce à ces techniques de " dissection " du noyau nous avons pu montrer qu'une enzyme, la PI-kinase est retrouvée associée à la matrice périphérique alors que trois autres enzymes; PIP-kinase, DAG-kinase et PLC sont retrouvées dans la matrice interne [13] (voir figure 1). Cette localisation différentielle, retrouvée dans les deux modèles d'étude (hépatocytes et fibroblastes au repos), suggère un rôle des matrices nucléaires dans la compartimentalisation de ces enzymes du métabolisme des phosphoinositides dans le noyau.

Quel peut être le rôle du cycle des phosphoinositides dans le noyau?

Plusieurs auteurs ont montré que la PKC pouvait phosphoryler les lamines sous l'effet d'ester de phorbol [16]. La phosphorylation des lamines par la p34cdc2 et d'autres kinases dont proba- blement la PKC, entraîne leur dépolymérisation, phénomène très important au moment de la mitose. D'autre part, un rôle de la PKC dans le transfert nucléocytoplasmique de l'ARNm a également été suggéré [17]. Un lien possible entre ces observations et l'activation d'une PLC nucléaire est apporté par la mise en évidence d'un parallélisme entre la production de DAG nucléaires et la translocation d'une PKC vers le noyau de fibroblastes Swiss 3T3 stimulés par l'IGF1 [4]. Cependant, si la PKC est transloquéc à l'intérieur du noyau, elle doit passer à travers les pores nucléaires ; or aucune séquence de translocation nucléaire n'a été identifiée dans les PKC connues et on ignore dans quelle mesure les DAG nucléaires pourraient intervenir?

D'autres rôles sont envisageables. En effet, il a été montré récemment qu'un pool de $\mathrm{Ca}^{2+}$ sensible à l'inositol

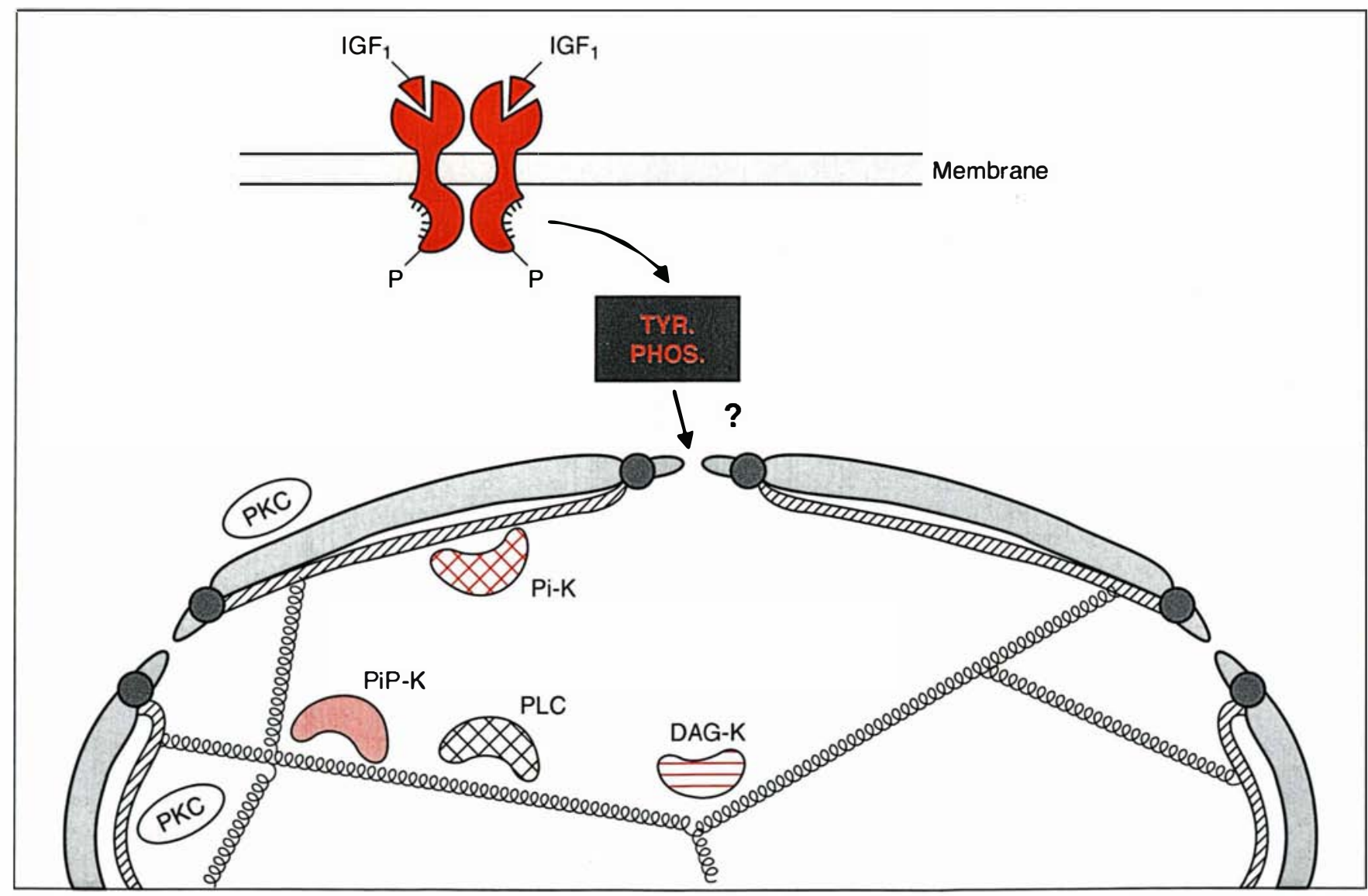

Figure 1. Représentation schématique des enzymes du métabolisme des phosphoinositides dans le noyau. Abréviations : IGF1, insulin growth factor ; TYR. PHOSP., phosphorylation sur résidus de tyrosine; PiK, phosphatidylinositolkinase ; PiP-K, phosphatidylinositolphosphate kinase; DAG-K, diacylglycérol-kinase; $P L C$, phospholipase $C$; $P K C$, protéine kinase $C$. 
1,4,5 trisphosphate peut exister dans les noyaux d'hépatocytes de rat [18]. De plus, un effet activateur de l'inositol, 1,4 bisphosphate et du PI4P sur l'ADN polymérase $\alpha$ a été mesuré in vitro [19], indiquant un rôle possible des inositol phosphates et des inositol lipides dans le métabolisme des acides nucléiques.

Bien que ces enzymes soient retrouvées dans le noyau de cellules au repos, une autre question importante s'adresse à la régulation des enzymes du métabolisme des phosphoinositides à l'intérieur du noyau : y sont-elles "transportées" en plus grande quantité au moment de la stimulation cellulaire, ou bien sontelles activées in situ ? De mĉme, le problème de "l'approvisionnement " en $\mathrm{PI}$, précurseurs des $\mathrm{PIP}_{2}$, soulève plusieurs questions : le cycle est-il complet dans le noyau avec une synthèse de novo de PI à partir d'acide phosphatidique et de cytidine monophosphate, ou bien le PI, dont la synthèse est classiquement envisagée dans le réticulum endoplasmique, est-il transporté par une protéine de transport des lipides, ce qui a déjà été suggéré [20] ? Comme nous le voyons, plusieurs hypothèses peuvent ĉtre proposécs mais ce domaine de recherche n'en est qu'à ses balbutiements.

Quoi qu'il en soit, le fait qu'un récepteur membranaire à activité tyrosine kinase intrinsèque (le récepteur à l'IGF1) soit capable de stimuler le cycle des phosphoinositides dans le noyau suggère l'intervention d'une cascade de phosphorylations par des kinases relais. Dans ce contexte, il faut noter qu'une liaison à l'ADN de la tyrosine kinase c-Abl a été récemment décrite [21]. Une observation importante est que l'IGF1 entraîne la phosphorylation spécifique sur des résidus de tyrosine de protéines nucléaires avec des cinétiques comparables à celle observée pour la production de DAG dans le noyau $[4,12]$. S'agit-il d'un phénomène particulier à l'IGF1 ou d'un mécanisme général rencontré pour tous les récepteurs à activité tyrosine kinase? Gageons qu'une meilleure compréhension de ces mécanismes verra le jour dans les mois ou les années à venir. Les données décrites ci-dessus permettent d'entrevoir le rôle que pourrait jouer les inositol lipides et leurs dérivés dans le noyau. Cepen- dant de nombreuses questions se posent, illustrant l'ampleur de ce nouveau domaine de recherche

Bernard Payrastre, P.M.P. Van Bergen en Henegouwen*, Hugues Chap Inserm U. 326, hôpital Purpan, 31059 Toulouse, France.

* Department of Molecular Cell Biology, University of Utrecht, 3584 CH Utrecht, The Netherlands.

\section{TIRÉS A PART}

\section{B. Payrastrc.}

\section{RÉFÉRENCES}

1. Berridge MJ. Inositol trisphosphate and diacylglyccrol : two interacting second messengers. Annu Rev Biochem 1987; 56 : 159-93.

2. Payrastre B, Plantavid M, Chap H. Phosphoinositide et transduction du signal. Regard sur la Biochimie 1992; 1: 44-9.

3. Payrastre B, van Bergen en Henegouwen PMP, Breton $M$, et al. Phosphoinositide kinase, diacylglyccrol kinase and phospholipase $\mathrm{C}$ activitics associated to the cytoskcleton. Effect of cpidermal growth factor. J Cell Biol $1991 ; 115: 121-8$.

4. Divecha N, Banfic H, Irvine RF. The polyphosphoinositide cycle exists in the nuclei of Swiss 3T3 cells under the control of a receptor (for IGF-1) in the plasma membrane, and stimulation of the cycle increases nuclear diacylglyccrol and apparently induces translocation of protcin kinase $\mathrm{C}$ to the nuclcus. EMBO J 1991; 10 : 3207-14.

5. Smith CD, Wells WW. Phosphorylation of rat liver nuclear envelopes J Biol Chem 1983 ; 258 : 9368-73

6. Cocco L, Gilmour RS, Ognibene A, Letcher AJ, Manzoli FA, Irvine RF. Synthesis of polyphosphoinositides in nuclei of Friend cells. Biochem J 1987 ; 248 : 765-70.

7. Cocco L, Martclli AM, Gilmour RS, Ognibenc A, Manzoli FA, Irvinc RF. Rapid changes in phospholipid metabolism in the nuclci of Swiss 3T3 cells induced by treatment of the cells with insulin-like growth factor I. Biochem Biophys Res Commun 1988; 154: 1266-72.
8. Capitani S, Bertagnolo V, Mazzoni M, et al. Lipid phosphorylation in isolated rat liver nuclei. Synthesis of polyphosphoinositides at subnuclear level. FEBS Lett 1989 ; 254 : 194-8.

9. Hocevar BA, Fields AP. Selective translocation of BII-proteine kinase $\mathrm{C}$ to the nucleus of human promyclocytic (HL60) leukemia cclls. J Biol Chem 1991; 266 : 28-33.

10. Jindal HK, Chancy WG, Anderson CW Davis RG, Vishwanatha JK. The proteintyrosine kinase substrate, calpactin I heavy chain ( $p 36$ ), is part of the primer recognition protein complex that interact with DNA polymerase $\alpha$. J Biol Chem 1991; 266 : 5169-76.

11. Raynal P, van Bergen en Henegouwen PMPO, Hullin F, et al. Morphological and biochemical evidence for partial nuclear localization of annexin 1 in endothelial cells. Biochem Biophys Res Commun 1992 (sous presse).

12. Ocmar BS, Law NM Rosenzwcig SA Insulin-like growth factor-1 induces tyrosyl phosphorylation of nuclcar protcins. J Biol Chem 1991; $266: 24241-4$.

13. Payrastre B, Nicvers $M$, Boonstra J, Breton $\mathrm{M}$, Verkleij $\mathrm{AJ}$, van Bergen en Hencgouwen PMP. A differential location of phosphoinositide kinases, diacylglycerol kinase and phospholipase C in the nuclear matrix. J Biol Chem 1992 ; 267 : 5078-84

14. De Jong L, van Dricl R, Stuurman N, Meijne AML, van Renswoude J. Principles of nuclear organization Int Rep Cell Biol 1990 ; $14: 1051 \cdot 74$.

15. Berczncy R. The nuclear matrix : a heuristic model for investigating genomic organization and function in the ccll nucleus. $J$ Cell Biochem 1991 ; 47 : 109-23.

16. Hornbeck P, Huang KP, Paul WE. Lamin $B$ is rapidly phosphorylated in lymphocytes after activation of protein kinase C. Prac Nall Acad Sci USA 1988 ; 85 : 2279-83.

17. Schröder HC, Rottmann M, Weger R, Bachmann M, Dorn A, Miller WEG. Studics on protein kinase involved in regulation of nuclcocytoplasmic mRNA transport. Biochem J 1988; 252: 777-90.

18. Malviya AN, Roque P, Yincendon G. Stercospecific inositol $1,4,5-[32 \mathrm{p}]$ trisphosphate binding to isolated rat liver nuclei : cvidence for inositol trisphosphate receptormediated calcium relcase from the nuclcus. Proc Natl Acad Sci USA 1990; 87 : 9270-4.

19. Sylvia V, Curtin G, Norman J, Stee J, Busbec D. Activation of a low specific activity form of DNA polymerase $\alpha$ by inositol 1,4 bisphosphatc. Cell $1988 ; 54$ : 651-8.

20. Capitani S, Helms JB, Mazzoni M, at al. Uptake and phosphorylation of phosphatidylinositol by rat liver nuclei. Role of phosphatidylinositol transfer protcin. Biochim Biophys Acta 1990 ; 1044 : 193-200.

21. Kipreos ET, Wang JYJ. Ccll cycleregulated binding of c-Abl tyrosinc kinase to DNA. Science $1992 ; 256: 382-5$. 